\title{
População em Situação de Rua: o Papel da Educação Médica ante a Redução de Iniquidades
}

\author{
The Homeless Population: the Role of Medical Education to Reducing Health Disparities
}

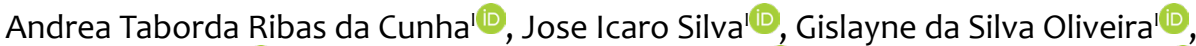

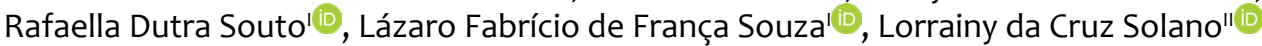

\begin{abstract}
RESUMO
Introdução: Compreendendo a abordagem holística de saúde e considerando as desigualdades brasileiras, infere-se a discrepância dos estados de saúde entre grupos sociais, sobretudo no que concerne à população em situação de rua (PSR). Essas pessoas lidam com o desamparo institucionalizado, e a pandemia da Covid-19 expôs ainda mais essa situação. Quanto à formação dos profissionais de saúde, torna-se fundamental a abordagem do tema.

Relato de Experiência: Com base na inclusão de experiências de docentes que articulavam ações de integração ensino-serviço com a equipe do Consultório na Rua de Mossoró, bem como a abordagem de temas relacionados às populações mais vulneráveis em eixo longitudinal ao curso, despertou-se o interesse dos discentes do curso de Medicina da Ufersa para ações direcionadas à PSR. Destarte, surge, em 2019, a ação "Saúde nas Ruas", em parceria com o Consultório na Rua, que fornece suporte à PSR em diversos aspectos, com a participação dos alunos do curso de Medicina. A ação, proposta a partir de iniciativa dos próprios alunos, marcou o início do planejamento da inclusão do atendimento a essa população no programa do internato do curso, bem como a criação de um projeto de extensão longitudinal para ações voltadas à PSR. Nesse processo, os discentes puderam imergir na realidade dessa população, e os usuários tiveram autonomia para compartilhar suas experiências e dores.

Discussão: Mediante uma formação acadêmica que visa romper com o modelo tecnicista, os estudantes possibilitaram voz à PSR e, compreendendo essas pessoas como sujeitos do processo, refletiram sobre o agir médico na transformação da realidade em que se inserem.

Conclusão: O corpo acadêmico participante potencializou em si a empatia e o desejo por uma saúde pública democrática e acessível. Ficou evidente ainda a imprescindibilidade de trabalhar temas específicos sobre populações vulneráveis, com o objetivo de fortalecer a atuação médica como forma de garantir o direito à saúde.
\end{abstract}

Palavras-chave: Pessoas em Situação de Rua; Empatia; Integralidade em Saúde; Populações Vulneráveis; Educação Médica.

\begin{abstract}
Introduction: A clear discrepancy in the health status between social groups in Brazil, particularly the homeless population (HP), can be inferred from the apparent inequalities when considering health from a holistic perspective. The homeless have to deal with institutionalized abandonment, and the COVID-19 pandemic has accentuated this issue. Addressing this theme has become an essential element of the training of health professionals.

Experience Report: The interest of medicine students at UFERSA in actions directed at the HP was triggered by teachers' experiences of coordinating service-learning integration actions with the street health care unit in Mossoró, and tackling issues related to the most vulnerable groups throughout the course. In 2019 the "Health on the Streets" action is therefore launched in partnership with the street health care unit, providing support to the HP in several aspects, and involving the participation of the medicine students. The action, proposed based on the students' own initiative, marked the start of the planning for including care for this group in the internship program of the course, as well as the creation of a longitudinal community outreach project for actions with the HP. The students had the opportunity to immerse themselves in the reality of this population, while the users had the autonomy to share their experiences and sufferings.
\end{abstract}

Discussion: Following an academic method that aims to break away from the technicist model, the students gave voice to the HP and, understanding these people as subjects of the process, reflected on the role of medicine in transforming their reality.

Conclusion: The academics who participated in this project enhanced their empathy and desire for democratic and accessible public health. It also became evident that tackling specific issues regarding vulnerable groups is indispensable, with a view to strengthening the role of the physician in guaranteeing the right to health.

Keywords: Homeless Persons; Empathy; Comprehensive Health Care; Vulnerable Groups; Medical Education.

'Universidade Federal Rural do Semi-Árido, Mossoró, Rio Grande do Norte, Brasil.

"Departamento Educação em Saúde da Prefeitura Municipal de Mossoró, Mossoró, Rio Grande do Norte, Brasil.

Correspondência

Andrea Taborda Ribas da Cunha.

Avenida Vingt Rosado, 1095, Costa e Silva, Mossoró, RN, Brasil. CEP: 59625-625.

E-mail: andrea.taborda@ufersa.edu.br

Recebido em 10/08/20; Aceito em 26/08/20 


\section{INTRODUÇÃO}

A $8^{a}$ Conferência Nacional em Saúde, realizada em 1986, trouxe consigo uma definição holística acerca do conceito de saúde1, a qual seria resultado da interação entre fatores habitacionais, educacionais, econômicos, ambientais, de lazer, de acesso aos serviços de saúde, entre outros² ${ }^{2}$. Considerando que esses determinantes se distribuem desigualmente na população brasileira, infere-se que o estado de saúde, por conseguinte, também seja discrepante entre os grupos sociais, especialmente no que tange à população em situação de rua (PSR).

No Brasil, atualmente, a PSR corresponde a cerca de 101 mil pessoas 3 . São indivíduos cujos direitos básicos, especialmente sociais - saúde, educação, segurança, trabalho, moradia e seguridade social ${ }^{4}$-, são cerceados ou negados. Com efeito, tais pessoas são relegadas à sorte que a rua lhes reservar, pois cria-se o mito de que elas são desqualificadas e estão nessa situação por escolha ${ }^{4}$, o que produz pouca ou nenhuma empatia da sociedade em relação a esse grupo e leva as próprias instituições, às quais caberia o papel de fonte de amparo, a perpetuar as desigualdades e privar essa população de dignidade humana.

O Estado, ao reconhecer sua falha na efetivação de direitos da PSR, instituiu, pelo Decreto $n^{\circ} 7.053$, de 23 de dezembro de 2009, a Política Nacional para a População em Situação de Rua, a qual tem por fito promover, por meio de políticas públicas, o acesso amplo, simplificado e seguro à cidadania5. Assim, entende-se que todas as esferas da sociedade e seus respectivos profissionais têm a missão de atuar para reduzir as desigualdades às quais a PSR é submetida e legar-lhe autonomia e protagonismo em sua história de vida.

Diante disso, é fundamental apontar o Sistema Único de Saúde (SUS) como ferramenta essencial à garantia do acesso à saúde por essa população, muito embora a atual disposição seja, por diversas vezes, ineficiente. Esse fato se comprova a partir de dois comportamentos principais, quais sejam: a percepção de profissionais da saúde que enxergam as pessoas em situação de rua como indivíduos com patologias sociais e, por isso, creem que não lhes cabe a responsabilidade de ajudá-las; e o comodismo desses profissionais em acreditar que é dever dessa população buscar os serviços de saúde, ignorando o despreparo dos ambientes institucionalizados para tratar as suas especificidades, assim como as transgressões que ocorrem nesses locais por meio da exigência da documentação, da restrição no atendimento de demanda espontânea e do preconceito².

Sob essa perspectiva, destaca-se que, apesar da constante atualização de currículo, o modelo hegemônico dos cursos de Medicina se mantém conteudista e organizado de maneira isolada e compartimentada, adotando métodos de avaliação que mensuram o acúmulo de informações técnicocientíficas padronizadas. O ensino tecnicista, dessa forma, foi responsável por favorecer a dissociação entre individual e coletivo, público e privado, biológico e social, curativo e preventivo ${ }^{6}$. Nesse ínterim, as novas Diretrizes Curriculares Nacionais do Curso de Graduação em Medicina (DCN) de 2014, conquanto ainda deficitárias, foram formuladas com o objetivo de mitigar essa problemática e explicitam o compromisso almejado das universidades de formar profissionais humanizados, generalistas, críticos e reflexivos, dotados de senso de responsabilidade social e que atuem como articuladores para políticas de atenção integral à saúde?

Nesse liame, a Universidade Federal Rural do SemiÁrido (Ufersa) construiu o Projeto Pedagógico do Curso (PPC) de Medicina centrado nas DCN, com o objetivo de formar profissionais capazes de trabalhar com populações nos seus mais variados contextos, de modo a possibilitar que o médico formado compreenda e contemple a plenitude de cada ser humano ${ }^{8}$. $O$ curso é baseado em metodologias ativas de ensino e aprendizagem, centradas no aluno, cuja condução integrativa dos módulos é feita pelo método aprendizagem baseada em problemas (problem based learning - PBL), além de utilizar outras metodologias associadas que promovem a reflexão, como narrativas e trabalhos com problematização. Desse modo, a universidade busca trabalhar, longitudinalmente, nos componentes modulares e optativos do curso, a temática de populações vulneráveis e grupos invisibilizados socialmente, de modo a possibilitar o desenvolvimento de empatia e a ampliação do conhecimento dos médicos em formação sobre o assunto, evidenciando, dessa maneira, a sensibilidade da graduação em estimular o senso crítico dos estudantes acerca dessas questões.

Por conseguinte, este relato reflexivo tem por objetivo compartilhar a experiência de alunos do primeiro ano do curso de Medicina da Ufersa ante a promoção de ações para a PSR. Tais atividades buscaram uma abordagem integral de cada indivíduo e do grupo como um todo, de forma a notabilizar o papel da universidade na construção do olhar crítico e equânime voltado às populações vulnerabilizadas desde os períodos iniciais ${ }^{9}$, bem como a responsabilidade social da escola médica perante o cenário local.

\section{RELATO DE EXPERIÊNCIA}

O município de Mossoró possui uma equipe de Consultório na Rua que conta, na modalidade proposta, com enfermeira, psicóloga, assistente social e dois agentes sociais. Os componentes dessa equipe têm um ótimo vínculo com os usuários que atendem, entretanto ainda manifestam um cuidado bem fragmentado e uma dificuldade de articulação com a rede, principalmente de atenção básica. Tal fato torna a 
assistência pouco efetiva em termos de cuidados longitudinais, mapeamento de agravos e articulação intersetorial com outras esferas do município.

A partir de 2018, alguns docentes do eixo de atenção primária à saúde do curso de Medicina da Ufersa, em parceria com as residências em saúde da Universidade do Estado do Rio Grande do Norte (Uern), começaram a acompanhar quinzenalmente as ações da equipe. Iniciaram-se, assim, a articulação de momentos integrados de planejamento e sala de situação e o delineamento de um cuidado longitudinal.

Com essa articulação, as ações passaram a se projetar dentro do currículo da graduação médica. Nesse sentido, o curso de Medicina em questão trabalha com o conceito de competência, buscando proporcionar a aprendizagem baseada em problemas como seu eixo norteador. Aliar a realidade local e as demandas e necessidades em saúde da população aos trabalhos dos objetivos de aprendizagem propostos é o fio condutor dos processos de ensino e aprendizagem do curso. Além disso, existe um eixo longitudinal que trabalha temas como empatia, comunicação, racismo, direitos humanos, desigualdades, entre outros, que complementam a formação médica e as competências colaborativas. Assim, em virtude da abordagem dos temas que vinham sendo trabalhados e exemplificados durante atividades dos eixos, muitos alunos despertaram maior sensibilidade para a temática. Dessa feita, os estudantes do curso, desde os primeiros períodos, desenvolvem interesse e liderança em coordenar ações voltadas para as populações invisibilizadas, como a PSR.

Tal interesse se expressa, por exemplo, na solicitação, por alunos ainda iniciantes do curso, de acompanhamento nos dias de atendimento e planejamento com a equipe do Consultório na Rua, em interlocução com os docentes. À vista disso, mediante pactuação com a equipe de saúde e com a população acompanhada, os alunos que almejavam construir um projeto de extensão com enfoque no trabalho com a PSR foram acolhidos. A priori, organizou-se uma grande iniciativa, a ação "Saúde nas Ruas", que foi planejada por um grupo de estudantes de Medicina que à época cursavam o primeiro período. A ação teve o intento de oferecer à PSR um momento de integralidade por meio do acesso aos serviços de saúde de forma contextualizada e acessível e que possibilitasse espaços de educação em saúde e autocuidado, além de introduzir a presença dos alunos nesse espaço já articulado. O evento foi arquitetado com a equipe de saúde do Consultório na Rua, bem como com as residências em saúde da Uern, e ocorreu em outubro de 2019, um ano após o início das primeiras atividades de integração ensino-serviço de cuidados destinados à PSR.

Inicialmente, foram propostas leituras sobre o tema e problematização sobre o perfil e os agravos aos quais essa população estava mais exposta. Optou-se pela montagem de diferentes estações no centro da cidade: infecções sexualmente transmissíveis (ISTs), saúde mental e alcoolismo, vacinação, testes rápidos, consulta com profissionais de saúde, primeiros socorros, higiene básica, alimentação e recebimento de doações de lençóis, roupas e calçados. Assim, realizou-se uma ação composta por profissionais e acadêmicos de diversas áreas, de modo a estimular neles a autonomia e a corresponsabilização, no sentido de garantir a essa população seus direitos básicos, especialmente no que concerne ao direito à saúde.

A ação ocorreu no adro da igreja catedral do município, zona que concentra maior parte da PSR de Mossoró e que já era ponto de atuação do Consultório na Rua, facilitando, então, a captação do público. Nesse ínterim, no dia e na hora em que a PSR já estava habituada às ações do Consultório na Rua, os acadêmicos das áreas supracitadas também compareceram ao local, no qual montaram as estações e fizeram a acolhida das pessoas nos arredores. Para a captação, utilizou-se a estratégia de convite mais íntimo, que consiste em conversar verdadeiramente com a pessoa e não apenas lhe transmitir uma informação, de modo que ela se sinta elemento crucial para o momento.

Mediante a aceitação, a pessoa transitaria no circuito de ações propostas, participando das estações que desejasse. As rodas de conversa partiram da perspectiva da Educação Popular em Saúde, sempre promovendo os assuntos e as discussões a partir do olhar do usuário. As ações de testagem contaram também com aconselhamento da equipe de saúde, bem como as de atendimento contaram com a interconsulta entre profissionais de diferentes áreas, promovendo assim um olhar ampliado da saúde e a visualização da proposta de um trabalho interdisciplinar aos discentes participantes.

$\mathrm{Na}$ estação de ISTs, fez-se uma roda de conversa e utilizaram-se álbuns seriados disponibilizados por unidade básica de (UBS) da cidade. Buscou-se abordar a importância do uso do preservativo, formas de contágio e sintomas característicos de ISTs, como sífilis, HIV/Aids e hepatites, e como os usuários se sentiam sobre esses assuntos. Nessa roda, percebeu-se, essencialmente, o desejo que tinham de compartilhar suas experiências na área da saúde, não necessariamente relacionadas às ISTs, mas, principalmente, evidenciando o descaso com o qual eram tratados.

No desenvolvimento das conversas, surgiram relatos como a proibição da realização de exames em instituições públicas em virtude da vestimenta que a pessoa utilizava, além de humilhação e constrangimento promovidos pelos próprios funcionários. Essas falas evidenciaram o quanto a atuação, ainda na qualidade de acadêmicos, pode ser significativa e transformadora. Os 
relatos dos participantes retrataram o quão raro e importante era ser "tratado como gente" e verdadeiramente ouvido, o que impactou fortemente todos os participantes.

A estação de saúde mental e alcoolismo, por sua vez, deu-se por meio de oficinas, a partir das quais, todos sentados, em roda, no chão, relataram como se sentiam e suas experiências com alcoolismo. Esse modelo foi escolhido para que os participantes se sentissem mais à vontade, de modo a quebrar a hierarquia entre acadêmicos, profissionais e usuários. Percebeu-se, outrossim, amplo envolvimento e interesse dos participantes, pois a maioria foi bastante ativa ao expor as próprias dores e viver a experiência com emoção e deleite.

A estação de primeiros socorros mostrou-se também bastante interessante. Os assuntos abordados nasceram das demandas apresentadas: o que fazer caso um colega tenha convulsão, abordagem sobre feridas e acidentes, entre outros. Por fim, distribui-se um fôlder, de autoria dos discentes, que continha a temática explanada com imagens de associação. Revelou-se, novamente, a partir dos diálogos, o quanto aquelas pessoas se sentiam à margem da sociedade por conta da naturalização da transgressão e da privação de seus direitos, o que foi evidenciado por olhares carentes de autoestima, de cuidado e de tratamento humanizado.

Na estação de higiene e cuidados pessoais, os discentes optaram por falar da importância do banho sempre que possível, do cuidado com o contato com fezes e urina e da atenção para a saúde bucal. Nesse âmbito, o que brotou aos olhos dos idealizadores também foi de amplo impacto: pareceu muito dissonante indicar comportamentos que sabidamente não dependiam exclusivamente da vontade dos participantes, mas eram, em maior parte, resultado das iniquidades às quais estavam sujeitos. Buscou-se, portanto, aumentar a autonomia dos participantes nesses cuidados, e, para tal, estabeleceramse parcerias com as empresas da cidade e com o próprio município, no intuito de montar kits de higiene básica para serem distribuídos aos participantes.

Por fim, houve entrega de arrecadações realizadas pelos discentes, com a distribuição de roupas, lençóis e calçados de forma equânime. Além disso, a ação contou com a disponibilização de refeições aos participantes, as quais foram custeadas pela prefeitura.

Conseguiu-se alcançar, por intermédio dessa ação, cerca de 30 pessoas em situação de rua, e o feedback foi feito por meio do diálogo com os participantes. Os discentes relataram que a ação foi muito positiva no contexto da PSR, principalmente porque dispuseram de "seus olhos e ouvidos" para realmente perceberem essa população e escutá-la de igual para igual. Os usuários se sentiram à vontade para falar de seus problemas, compartilhar suas experiências e expor suas dores, permitindo que os estudantes pudessem conversar com cada um de forma direcionada e atenta.

Outras ações foram formuladas a partir desse contexto vivenciado: a proposta anterior de extensão foi retomada e articulou-se a inclusão da participação das ações do Consultório na Rua nas atividades do internato. Entretanto, a chegada da pandemia retardou a continuidade das ações presenciais dos alunos de graduação, mas eles se mantiveram em campanhas de auxílio de arrecadação de insumos de higiene e alimentos e confecção de materiais para higienização de mãos.

Houve também sensibilidade por parte da gestão da instituição e do corpo docente em exercer seu papel de responsabilidade social, mesmo com as atividades acadêmicas paradas pela pandemia. De comum acordo, houve a inserção dos docentes em atividades assistenciais à população de rua no Abrigo Municipal Provisório criado para a PSR na pandemia, assim como foram mantidas atividades ambulatoriais de pré-natal para gestantes em situação de rua e testagem da população por teste rápido imunocromatográfico adquirido pela universidade. Além dessas ações, o corpo docente do curso atuou, ativamente, como apoio institucional na reorganização da rede municipal de saúde para o enfrentamento da pandemia elaborando protocolos e notas técnicas para colaborar com a produção dos serviços de saúde.

Outros cursos da instituição também se articularam para atender às demandas dessa população, como o Núcleo de Educação a Distância (NEAD) que, em parceria com o curso de Medicina e a gestão municipal, proporcionou formação EaD sobre assistência à PSR e lives com lideranças do Movimento Nacional Pop Rua. Houve, ainda, participação dos cursos de Ciência e Tecnologia por meio da produção de materiais de proteção para as equipes atuantes com a PSR. Tais iniciativas fortaleceram os laços com a equipe de saúde responsável pelo atendimento a essa população e ampliaram as relações com setores diversos, como estruturas e profissionais da Ação Social do município.

Tais ações têm demonstrado que os serviços deixaram de ser um mero cenário e transformaram-se em parceiros na qualificação para o atendimento em saúde, bem como a prática interprofissional tornou-se um norte para a solução dos problemas.

\section{DISCUSSÃO}

Faz-se imprescindível ressaltar os aspectos da graduação de Medicina da Ufersa que incentivaram os estudantes a se sentir desenvoltos ao realizarem as atividades com grupos vulneráveis, mormente com a PSR. Entre eles, destaca-se a integração existente entre os eixos modulares e 
optativos do curso e, consequentemente, em suas avaliações. Dessa forma, atua-se na tentativa romper a dicotomia entre os conhecimentos sociais e biológicos, a qual é apontada por estudos como um dos maiores problemas nas graduações da saúde, na medida em que eclipsa o aprendizado e a formação integral do estudante de Medicina ao perpetuar a fragmentação entre o pensar/fazer e o individual/coletivo ${ }^{10}$.

Corroborando o supracitado, desde os primeiros períodos do curso, os discentes são instruídos a estabelecer contato com a PSR, por meio da participação em projetos e ações voluntários. Nesse sentido, a compreensão do indivíduo em todo o seu contexto biopsicossocial faz desenvolver nos estudantes, ainda que trilhando seus passos iniciais na medicina, um perfil de maior responsabilização social, contribuindo para formar profissionais mais humanizados.

Em acréscimo, observou-se a importância da diversificação do cenário de aprendizagem no tocante ao tratamento da PSR. A ruptura do modelo tradicional de ensino dos ambientes institucionalizados de saúde possibilitou aos estudantes expandir os seus horizontes de conhecimentos acerca dessa comunidade que, pelos motivos mencionados, não frequentam hospitais e UBSs. Esse fator reitera a literatura que assinala a necessidade de o ensino médico transpor o ambiente acadêmico e o dever do profissional médico de se adequar às demandas das diferentes comunidades ${ }^{10}$.

Desse modo, a Ufersa cumpre uma função importante ao instruir que os estudantes reflitam acerca desse problema e proponham formas viáveis de atuar para sua superação. Logo, notabiliza-se a indispensabilidade de a graduação fomentar o senso crítico dos discentes, haja vista que, consoante Miranda ${ }^{11}$, urge a necessidade de que os alunos sejam capazes não apenas de entender como ocorrem as problemáticas sociais, mas também como o médico deve atuar alterando a realidade na qual está inserido, visando efetivar o direito à saúde de populações vulneráveis.

Outro aspecto a ser sublinhado é a necessidade da voz ativa das pessoas que se encontram em situação de rua. Salienta-se que, para entender melhor as especificidades do processo saúde-doença, foi necessário que os médicos em formação exercessem o diálogo com essa população. Nesse diapasão, a literatura alerta para a importância de essas pessoas serem enxergadas não apenas como objetos de intervenção, mas também como sujeitos da relação, de modo que essa população participe ativamente do processo de melhoria de suas condições de vida' ${ }^{12}$, sobretudo num contexto desprovido de políticas intersetoriais.

Tendo em vista todos esses fatores, é importante correlacionar o status da PSR com a pandemia vivenciada. Segundo Aguiar et al. $^{13}$, depreende-se que, por possuir limitações e fragilidades, a PSR tem seus agravos em saúde potencializados durante a pandemia do novo coronavírus. Assim, faz-se crucial elaborar estratégias com o intuito de assegurar que essa comunidade possua condições mínimas para a superação da doença. Para isso, estudantes e professores da graduação se inseriram em campanhas voltadas para essa população, objetivando o arrecadamento de insumos de higiene básica, alimentos e roupas. Destaca-se que houve a sensibilidade de informar acerca da vigente situação mundial, nacional e local e da prevenção contra o vírus, levando em consideração as suas vulnerabilidades, ao passo que muitos não possuem acesso a meios de comunicação de massa.

Convém ressaltar que, pelo fato de o curso de Medicina da Ufersa ainda ser recente na comunidade acadêmica, os estudantes observam pontos passíveis de aprimoramento, tais como a inserção de uma tutoria que trabalhe especificamente a temática da PSR. Um estudo realizado por To et al. ${ }^{14}$ aponta a primordialidade de se trabalharem casos que envolvam essa comunidade com o intuito de humanizar e familiarizar os estudantes com o assunto, bem como para promover o embasamento teórico necessário acerca das especificidades das pessoas que se encontram nessa situação.

Ademais, é importante que esse assunto seja inserido nas grades curriculares do curso de Medicina para que os tabus desse tema sejam quebrados, dado que pesquisas e produções científicas sobre a temática revelam a inaptidão do profissional médico em atender os pacientes da PSR, reforçando a opressão da qual essa comunidade é vítima ${ }^{15}$.

Desse modo, acreditamos que a graduação de Medicina da Ufersa consegue construir um profissional com maiores possibilidades à medida que cumpre o papel social de ser parte da rede local de saúde, garantindo um modelo responsável de formação comprometido com as necessidades de saúde da população no sistema local de saúde.

\section{CONCLUSÃO}

Os discentes e docentes ligados à graduação potencializaram a empatia e solidificaram em si o desejo por uma saúde pública democrática, de qualidade e acessível que a experiência ao longo do curso já havia semeado. Durante o momento, afirmamos, então, a essencialidade de um olhar voltado às minorias com capacidade crítica, reflexiva e transformadora, despertando senso de responsabilidade pela comunidade na qual nos inserimos.

Notamos ainda a imprescindibilidade de uma abordagem particular para essa população nas tutorias do curso destacado, fortalecendo, nos médicos generalistas a serem formados, a capacidade de atuação contextualizada e direcionada, permitindo, então, significativas transformações 
no que concerne à contribuição do SUS para a garantia do direito à saúde para a PSR.

Ademais, apesar do sucesso da ação em alcançar a PSR, percebemos alguns entraves na continuidade de mais edições do "Saúde nas Ruas", especialmente em virtude do cenário pandêmico vigente, que impede a reunião presencial de toda a equipe e a aglomeração de pessoas nos espaços públicos. Com efeito, o acesso direto ao público-alvo tal qual foi realizado em 2019 não se faz viável, sendo substituído por ações nas quais os acadêmicos atuam nos bastidores. Outrossim, foram encontradas limitações referentes à manutenção do contato com toda a equipe multidisciplinar atuante, dificultando uma programação contínua entre os acadêmicos e as parcerias, o que tem sido confrontado pela tentativa constante de trabalho conjunto entre os serviços. Com o propósito de sanar ao máximo as lacunas oriundas das condições atuais, entendemos que a utilização dos meios digitais para o aperfeiçoamento e a reorganização da equipe é uma solução exequível não só para a Ufersa, mas também para as outras escolas médicas.

Portanto, acreditamos que a graduação médica da Ufersa seja exitosa em formar profissionais mais habilitados no tocante ao tratamento das pessoas em situação de rua, a fim de que se construa uma medicina cada vez mais humana e capaz de fornecer vez e voz a essa população ainda tão negligenciada e estigmatizada no país. Nesse viés, notamos a necessidade de que ações semelhantes sejam reproduzidas nas escolas médicas do Brasil, objetivando construir acadêmicos e profissionais médicos que façam a diferença em sua realidade.

\section{CONTRIBUIÇÃO DOS AUTORES}

Andrea Taborda Ribas da Cunha, Jose Icaro Silva, Gislayne da Silva Oliveira e Rafaella Dutra Souto participaram da elaboração e execução da ação relatada, e da escrita e revisão do relato. Lázaro Fabrício de França Souza participou da revisão do relato. Lorrainy da Cruz Solano participou da ação relatada e da revisão do relato.

\section{CONFLITO DE INTERESSES}

Os autores declaram não haver conflito de interesses neste estudo.

\section{REFERÊNCIAS}

1. Ministério da Saúde. $8^{\circ}$ Conferência Nacional de Saúde. Brasília: Ministério da Saúde; 1986 [acesso em 8 ago 2020]. Disponível em: https:// bvsms.saude.gov.br/bvs/publicacoes/8 conferencia nacional saude relatorio_final.pdf.
2. da Cunha JVQ, Rodrigues M, organizadoras. Rua: aprendendo a contar. Brasília: Athalaia Gráfica; 2009 [acesso em 9 ago 2020]. Disponível em: https://www.mds.gov.br/webarquivos/publicacao/assistencia_social/ Livros/Rua_aprendendo_a_contar.pdf.

3. Natalino MAC. Estimativa da população em situação de rua no Brasil. Brasília: Ipea; 2016 [acesso em 8 ago 2020]. Disponível em: https://www. ipea.gov.br/portal/images/stories/PDFs/TDs/26102016td_2246.pdf.

4. Ministério Público do Distrito Federal e Território. Direitos das pessoas em situação de rua. Brasília: Ministério Público do Distrito Federal e Território; 2018 [acesso em 9 ago 2020]. Disponível em: https://www. mpdft.mp.br/portal/pdf/imprensa/cartilhas/cartilha_direitos_das pessoas_situacao_de_rua_mpdft.pdf.

5. Brasil. Política Nacional para a População em Situação de Rua. Brasília: Ministério da Mulher, da Família e dos Direitos Humanos [acesso em 9 ago 2020]. Disponível em: https://www.gov.br/mdh/pt-br/naveguepor-temas/populacao-em-situacao-de-rua/politica-nacional-para-apopulacao-em-situacao-de-rua.

6. de Mello ALSF, Moysés ST, Moyés SJ. A universidade promotora de saúde e as mudanças na formação profissional. Interface Comun Saúde Educ. 2010;14(34):683-92.

7. Brasil. Resolução $n^{\circ} 3$, de 20 de junho de 2014. Institui as Diretrizes Curriculares Nacionais do Curso de Graduação em Medicina e dá outras providências. Brasília; 2014 [acesso em 9 ago 2020]. Disponível em: http:// portal.mec.gov.br/cne/arquivos/pdf/Med.pdf.

8. Brasil. Projeto Pedagógico do Curso de Medicina: Campus Central Mossoró Mossoró: Universidade Federal Rural do Semi-Árido; 2016 [acesso em 30 ago 2020]. Disponível em: https://documentos.ufersa.edu.br/wp-content/ uploads/sites/79/2016/02/Projeto_Pedagogico_Medicina.pdf.

9. Pagani C, Schommer PC. O papel da universidade em iniciativas voltadas à informação, cidadania e accountability em Córdoba e São Paulo. Cadernos Gestão Pública e Cidadania. 2017;22(71):103-25 [acesso em 9 ago 2020]. Disponível em: http://dx.doi.org/10.1266o/cgpc.v22n71.63434.

10. Ceccim RB, Feuerwerker LCM. Changes in undergraduate education in the health professions from the perspective of comprehensive training. Cad Saúde Pública. 2004;20(5):1400-10 [acesso em 9 ago 2020]. Disponível em: http://www.scielo.br/scielo.php?script=sci_arttext\&pid=S0102-311X2 $004000500036 \&$ Ing=en\&nrm=iso\&tlng=pt.

11. Miranda MGO. Projeto político de formação do enfermeiro: contextos, textos, (re)construções [tese]. Natal: Universidade Federal do Rio Grande do Norte, 2010 [acesso em 9 ago 2020]. Disponível em: https:// repositorio.ufrn.br/jspui/handle/123456789/18311.

12. Pinheiro R, da Silva Junior AG, organizadores. Cidadania do cuidado: o universal e o comum na integralidade das ações de saúde. Rio de Janeiro: IMS/ Ueri, Cepesc; 2011 [acesso em 9 ago 2020]. Disponível em: www.lappis.org.br.

13. Aguiar A, Meireles P, Rebelo R, Barros H. Covid-19 e as pessoas em situação de sem-abrigo: ninguém pode ser deixado para trás. Da emergência de um novo vírus humano à disseminação global de uma nova doença - Doença por Coronavírus 2019. ResearchGate, 2020. [acesso em 9 ago 2020]. Disponível em: https://www.researchgate.net/ publication/340204725_COVID-19_e_as_pessoas_em_situacao_de_semabrigo_Ninguem_pode_ser_deixado_para_tras

14. To MJ, Macleod A, Hwang SW. Homelessness in the medical curriculum: an analysis of case-based learning content from one Canadian medical school. Teach Learn Med. 2016;28(1):35-40 [acesso em 9 ago 2020]. Disponível em: http://www.tandfonline.com/action/ journallnformation?journalCode=ht/m2o.

15. de Paiva IKS, Lira CDG, Justino JMR, Miranda MGDO, Saraiva AKDM. Direito à saúde da população em situação de rua: reflexões sobre a problemática. Ciênc. Saúde Colet. 2016;21(8): 2595-606 [acesso em 9 ago 2020]; Disponível em: http://www.scielo.br/scielo.php?script=sci_ arttext\&pid=S1413-81232016000802595\&lng=en\&nrm=iso\&tlng=pt. 